1 Smith AF, Smith JG. The economic burden of global blindness: a price too high! Brf Ophthalmol 1996;80:276-7.

2 Smith AF. Ophthalmic services research: towards increasing the role of economic and operations research methods in the delivery of eye care. $\mathrm{Br} \mathcal{F}$ Ophthalmol 1995;79:515-6.

3 Rosenthal AR, Thompson JR. Applications of epidemiological techniques. Eye 1995;9:671-3.

4 Wormald R. Assessing the prevalence of eye disease in the community. Eye 1995;9:674-6.
5 Desai P. Assessing ophthalmic services. Eye 1995;9:677-8.

6 Sommer A. Toward affordable, sustainable eye care. Int Ophthalmol 1995;18:287-92.

7 Tielsch JM, Sommer A, Katz J, Royall RM, Quigley HA, Javitt J. Racial variations in the prevalence of primary open-angle glaucoma: the Baltimore Eye Survey. $\mathcal{F} A M A 1991 ; 266: 369-74$.

8 Desai P. The National Cataract Surgery Survey: II. Clinical outcomes. Eye 1993:7:489-94.

\title{
Progression of diabetic retinopathy following cataract surgery: can it be prevented?
}

Diabetic patients have an increased risk of developing cataract. This risk is related to age, severity of retinopathy, duration of the disease and, possibly, systemic hypertension. ${ }^{12}$ Harding et al in 1992, using data from the Oxford Region in the UK, demonstrated that diabetes mellitus is associated with a fivefold increase in the risk of developing cataract. They further estimated that $11 \%$ of all cataracts in the UK were in diabetic patients. ${ }^{3}$ The Royal College of Ophthalmologists' audit of cataract surgery in 1991 revealed that $4 \%$ of all patients undergoing cataract surgery had diabetic retinopathy. Approximately 50 patients with diabetic retinopathy have cataract surgery each week in the UK. ${ }^{4}$ Cataract is very common after laser photocoagulation for proliferative diabetic retinopathy. Blankenship, in 1989, reviewed all the survivors of the original diabetic retinopathy study photocoagulation trial in 1976 and found that 13 out of 51 eyes had had cataract surgery in the 15 years after photocoagulation. ${ }^{5}$ In addition, posterior vitrectomy for vitreous haemorrhage and tractional retinal detachment is frequently followed by cataract.

Cataract surgery in diabetic patients, therefore, forms a significant part of every eye department's workload. It is obviously important that such patients are managed appropriately to minimise visual loss from progression of diabetic retinopathy.

Cataract surgery in diabetics with little or no retinopathy has the same good prognosis as cataract surgery in non-diabetics. ${ }^{6-8}$ However, in the presence of significant diabetic retinopathy the results can be disappointing. ${ }^{9-11}$ The paper by Henricsson et al in this issue of the BFO (p 789) demonstrates, for the first time, that it is possible, in the presence of diabetic retinopathy, to predict outcome. In this small, but carefully documented prospective study, $89 \%$ of eyes achieved $6 / 12(0.5)$ or better vision and maintained it for at least 18 months. One third of the eyes had non-proliferative diabetic retinopathy and almost a quarter had treated proliferative diabetic retinopathy.

Severe visual loss following cataract surgery in diabetics may be due to worsening macular oedema, continuing anterior and posterior segment proliferation, posterior capsule opacification, or unrelated events, such as retinal vein occlusion. Risk factors associated with worsening retinopathy after cataract surgery include pre-existing severe treated or untreated retinopathy, poor glycaemic control, increasing age, and planned or unplanned posterior capsule disruption.

Non-proliferative diabetic retinopathy can rapidly progress to severe diffuse macular oedema in the months following uncomplicated cataract extraction. Jaffe and Burton $^{12}$ and later Schatz et $a l^{13}$ emphasised that the retinopathy progressed rapidly in the operated eye compared with the fellow, control phakic eye. None of the patients documented in these reports had received any preoperative photocoagulation. Pollack et $a l^{9}{ }^{9}$ as well as Cunliffe $e t a l^{6}$ showed that macular oedema and neovascularisation can worsen after cataract extraction even with photocoagulation. Fortunately, only a small minority of diabetics with non-proliferative diabetic retinopathy develop severe persistent macular oedema and poor vision after cataract surgery. Pollack et al ${ }^{14}$ also demonstrated that $81 \%$ of eyes with pre-existing background retinopathy developed clinical cystoid macular oedema after uncomplicated cataract surgery compared with only $32 \%$ of eyes without background retinopathy. Menchini et al ${ }^{15}$ compared the incidence of cystoid macular oedema following cataract extraction in diabetics with no diabetic retinopathy with normal non-diabetic controls using fluorescein angiography. They found that the incidence of fluorescein angiographic macular oedema was similar at 30 days in both groups, but that $24.5 \%$ of the pseudophakic diabetic eyes still had macular oedema at 1 year compared with none of the non-diabetic pseudophakic controls. This suggests that the blood-retinal barrier is significantly impaired, even in diabetics with no retinopathy and that cataract surgery worsens this impairment.

Preoperative and early postoperative photocoagulation for macular oedema appears to reduce but not to eliminate the risk of visual loss. For this reason, careful preoperative assessment and regular follow up, if necessary using fluorescein angiography, are essential. Fluorescein angiography is particularly required to differentiate between pseudophakic cystoid macular oedema and worsening diabetic macular oedema which may require photocoagulation. ${ }^{9}$

Many patients, including those with diabetic retinopathy, may have very high expectations from cataract surgery. For this reason, patients with diabetic retinopathy and cataract need to be advised preoperatively that retinopathy and vision may worsen after cataract extraction. ${ }^{113}$

Neovascular glaucoma and rapidly progressive proliferative diabetic retinopathy can occur after extracapsular cataract surgery in treated and untreated proliferative diabetic retinopathy. ${ }^{6111617}$ Pollack et al ${ }^{9}$ described the rapid development of severe retinal ischaemia confirmed by fluorescein angiography in the 3 months following uncomplicated extracapsular cataract surgery. The visual results of extracapsular cataract surgery in treated proliferative diabetic retinopathy with maculopathy are frequently poor. However, good results have been reported in well treated proliferative diabetic retinopathy without maculopathy. ${ }^{616}$

Adequate panretinal laser photocoagulation is therefore essential if there is severe peripheral retinal ischaemia or early retinal neovascularisation. This photocoagulation should be applied preoperatively. If this is not possible it can be done peroperatively using the laser indirect 
ophthalmoscope or in the early postoperative period. Otherwise, a severe fibrinous uveitis with adhesions may prevent photocoagulation. Eyes with severe untreated retinopathy always have abnormally permeable iris vessels so a severe fibrinous uveitis may follow even minimal iris trauma in uncomplicated surgery. ${ }^{18}$

Age can be a useful predictor of outcome following cataract surgery in diabetics. Benson et al ${ }^{11}$ reported that $58 \%$ of patients under 63 achieved $6 / 12$ or better but only $38 \%$ of their patients over 64 years of age achieved this level.

Posterior capsular opacification is significantly more common in diabetics with retinopathy than in nondiabetics. Ionides et al stressed the importance of early capsulotomy to ensure an adequate retinal view. ${ }^{19}$

The visual results reported following cataract surgery in diabetics by Henricsson et al, on p 789, are significantly better than the results from equivalent series in the past: $89 \%$ of their patients achieved $6 / 12$ or better. This compares favourably with $67 \%$ in Cunliffe et al's series, ${ }^{6}$ $67 \%$ in Hykin et als series, ${ }^{16}$ and $48 \%$ in Benson et als report. $^{11}$

It is of particular interest that only one operated eye lost vision after surgery and this was due to a retinal vein occlusion. In two patients the visual acuity was worse in the operated eye compared with the phakic control fellow eye. Overall, however, there was no difference in the rate of progression of the retinopathy between the operated and unoperated eyes.

Most previous reports have stressed that retinopathy may progress rapidly in the pseudophakic eye compared with the control fellow phakic eye. Henricsson et al found that any progression of retinopathy appeared to be related to higher levels of haemoglobin $A_{l c}$ pre- and postoperatively and not to cataract surgery. Most patients in their study had reasonably good control of blood sugar with no patient having a haemoglobin $A_{1 c}$ greater than $9.1 \%$. Good glycaemic control and its beneficial effect on progression of retinopathy after cataract surgery has not been reported in the past.

In summary, the good results achieved in this paper appear to be due to the following factors: the cataract extraction was performed when indicated as part of a long term comprehensive diabetic care programme; there was careful long term control of blood sugar; there was appro- priate pre- and postoperative photocoagulation; and the decision to operate was based on detailed knowledge of the visual acuity and retinopathy over several years, thus reducing the likelihood of cataract surgery on eyes with poor visual potential.

Addenbrooke's Hospital NHS Trust,

D W FLANAGAN

Cambridge CB2 2QQ

1 Klein BEK, Klein R, Moss SE. Prevalence of cataracts in a population-based study persons with diabetic maculopathy. Ophthalmology 1985;92:1191-6.

2 Szmyd L, Schwartz MD. Association of systemic hypertension and diabetic mellitus with cataract extraction. A case control study. Ophthalmology 1989;96:1248-52.

3 Harding J, Egerton M, van Heyningen R, Harding RS. Diabetes, glaucoma, sex, and cataract: analysis of combined data from two case control studies. Brf Ophthalmol 1993;77:2-6.

4 Courtney P. The National Cataract Survey:1. Method and descriptive features. Eye 1992;6:487-92.

5 Blankenship W. 15 year Argon laser and xenon photocoagulation. Results of Bascom Palmer Eye Institute patients participating in the diabetic retinopathy study. Ophthalmology 1991;98:125-8.

6 Cunliffe IA, Flanagan DW, George NDL Aggarwaal RJ, Moore AT Extracapsular ctaract extraction with lens implantation in diabetics with Extracapsular ctaract extraction with lens implantation in diabetics with
and without proliferative diabetic retinopathy. Br f Ophthalmol 1991;75:9-

and 12.

7 Stratctsman BR, Pettit TH, Wheeler N, Miyamou W. Diabetes mellitus and intraocular lens implantation. Ophthalmology 1983;90:336-43.

8 Sebestyen JG. Intraocular lenses and diabetes mellitus. Am $\not$ Ophthalmol 1986;101:425-8.

9 Pollack A, Dotan S, Oliver M. Course of diabetic retinopathy following cataract surgery. Br $\mathcal{F}$ Ophthalmolol 1991;75:2-8.

10 Smith R. Diabetic retinopathy and cataract surgery. [Editorial] $\mathrm{Br} \mathfrak{g}$ Ophthalmol 1991;75:1.

11 Benson WE, Brown GC, Tasman W, NcNamara JA, Vander JF. Extracapsular cataract extraction with placement of a posterior chamber lens in patient with diabetic retinopathy. Ophthalmology 1993;100:730-8.

12 Jaffe GJ, Burton TC. Progression of non-proliferative diabetic retinopathy following cataract extraction. Arch Ophthalmol 1988;106:745-9.

13 Schatz H, Atienza D, McDonald R, Johnson RN. Severe diabetic retinopathy after cataract surgery. Am f Ophthalmol 1994;117:314-21.

14 Pollack A, Leiba H, Buckelman A, Oliver M. Cystoid macular oedema following cataract extraction in patients with diabetes. Br f Ophthalmol 1992; 76:221-4.

15 Menchini U, Bandello F, Brancato R, Camesasca FI, Galdini M. Cystoid macular oedema after extracapsular cataract extraction and intraocular lens implantation in diabetic patients with retinopathy. $B r f \mathcal{O}$ Ophthalmol 1993;77:208-10.

16 Hykin PG, Gregson RMC, Stevens JD Hamilton AM. Extracapsular cataract extraction in proliferative diabetic retinopathy. Ophthalmology 1993;100:394-9.

17 Sadiq SA, Chatterjee A, Vernon SA. Progression of diabetic retinopathy and rubeotic glaucoma following cataract surgery. Eye 1995;9:728-32.

18 Bandello F, Brancato R, Lattanzio R, Galdini M, Falcomata B. Relation between iridopathy and retinopathy in diabetes. Br f Ophthalmol 1994;78; 542-5.

19 Ionides A, Bowles JGF, Hykin PG, Rosa PH, Hamilton AM. Posterior capsule opacification following diabetic extracapsular cataract extraction. Eye 1994;8:535-7. 